\title{
Interférences
}

Ars scribendi

\section{Vie publique et vie privée dans les lettres de Cicéron à Terentia (Ad Familiares 14, 1-4)}

\section{François Guillaumont}

\section{(2) OpenEdition}

1 Journals

\section{Édition électronique}

URL : http://journals.openedition.org/interferences/5457

DOI : 10.4000/interferences.5457

ISSN : $1777-5485$

Éditeur

HiSoMA - Histoire et sources des Mondes antiques

\section{Référence électronique}

François Guillaumont, «Vie publique et vie privée dans les lettres de Cicéron à Terentia (Ad Familiares 14, 1-4) », Interférences [En ligne], 8 | 2015, mis en ligne le 30 octobre 2014, consulté le 15 septembre 2020. URL : http://journals.openedition.org/interferences/5457 ; DOI : https://doi.org/10.4000/ interferences. 5457

Ce document a été généré automatiquement le 15 septembre 2020.

Tous droits réservés 


\title{
Vie publique et vie privée dans les lettres de Cicéron à Terentia (Ad Familiares 14, 1-4)
}

\author{
François Guillaumont
}

1 Mis à part une lettre à Q. Metellus Nepos, l'un des consuls de 57, les lettres d'exil de Cicéron s'adressent à trois correspondants, qui sont tous trois des intimes : son ami Atticus, sa femme Terentia et son frère Quintus. Pour la période de l'exil, nous disposons de 27 lettres à Atticus (formant le livre 3 des Lettres à Atticus), 4 lettres à Terentia (adressées aussi aux enfants de Cicéron) et 2 lettres à Quintus. Il s'agit là des lettres conservées. Celles-ci font état d'autres lettres, qui ne sont pas parvenues jusqu'à nous ${ }^{1}$, et il est probable que Cicéron en avait écrit encore d'autres qui n'ont pas laissé de trace dans sa correspondance.

$2 \mathrm{Au}$ début de février 57, la correspondance s'interrompt pour environ sept mois et ne reprend qu'au milieu du mois de septembre, après le retour de Cicéron en Italie. Dans le cas d'Atticus, ce silence pourrait s'expliquer par le fait que les deux amis ont passé une partie de cette période ensemble, soit à Dyrrachium, soit dans le domaine d'Atticus à Buthrote, en Épire: il s'agit là d'une simple hypothèse, due à L.-A. Constans; nous n'avons pas la preuve d'un séjour de Cicéron avec Atticus ${ }^{2}$. De toute façon cette explication ne vaut pas dans le cas de Terentia et de Quintus: il est probable que Cicéron leur a adressé des lettres qui sont aujourd'hui perdues ${ }^{3}$.

3 Les quatre lettres à Terentia sont les plus anciennes des lettres de Cicéron à son épouse. La première a été écrite à Brindes le 29 avril 58 , le jour même où Cicéron quitte l'Italie et s'embarque pour Dyrrachium $\left(\right.$ Fam. 14, 4) ${ }^{4}$. La seconde lettre a été écrite le 5 octobre à Thessalonique, donc plus de cinq mois après la précédente (Fam.14, 2). Au début de cette lettre, Cicéron tente de justifier la brièveté de ses missives, dont Terentia s'était peut-être plainte. On peut donc supposer qu'entre avril et octobre il n'a envoyé que de courts billets qui n'ont pas été conservés. La troisième lettre a été remise à un courrier le 25 novembre à Dyrrachium mais, pour l'essentiel, elle a été écrite à Thessalonique $($ Fam. 14, 1). En effet, au mois de novembre, Cicéron quitte Thessalonique pour 
Dyrrachium. Deux raisons expliquent ce changement de résidence. Cicéron sait qu'un nouveau gouverneur va arriver à Thessalonique, capitale de la province de Macédoine : il s'agit de Pison, l'un des consuls de 58, qu'il considère comme l'un de ses pires ennemis. D'autre part, il se rend compte que la situation à Rome évolue favorablement et il souhaite se rapprocher de l'Italie. La durée du voyage étant évaluée à une quinzaine de jours ${ }^{5}$, la lettre à Terentia a pu être commencée autour du 10 novembre. Enfin la quatrième lettre a été remise le 29 novembre à Dyrrachium (Fam. 14, 3).

Pour chaque lettre à Terentia, nous possédons une lettre à Atticus envoyée le même jour. Cela n'a rien d'étonnant : quand Cicéron dispose d'un courrier, il en profite pour écrire à plusieurs correspondants. Ainsi, le 13 juin, il écrit à la fois à Atticus et à son frère Quintus ${ }^{6}$. En comparant les lettres écrites le même jour, nous pouvons étudier la manière dont Cicéron adapte le contenu et la forme de son message à la personnalité du destinataire.

5 Avant d'en venir à cette comparaison, il convient de procéder à un rappel biographique à propos de Terentia et de ses enfants. On ne sait pas exactement quand Cicéron et Terentia se sont mariés, même si les spécialistes sont d'accord pour placer le mariage entre 80 et $76^{7}$. Comme Cicéron a fait un long voyage d'études en Grèce et en Asie mineure entre 79 et 77 , l'hypothèse la plus vraisemblable, retenue notamment par Pierre Grimal, est celle qui place le mariage en 77 , au retour du voyage en Grèce ${ }^{8}$. Nous ne savons rien sur Terentius, le père de Terentia. Il était peut-être noble, en tout cas très riche, puisque, selon Plutarque, Terentia a apporté à son mari une dot considérable ${ }^{9}$. Elle possédait en outre diverses propriétés, dont il est parfois question dans les lettres de Cicéron ${ }^{10}$. Nous savons d'autre part qu'elle avait une demi-sœur, ou peut-être une cousine, Fabia, qui était Vestale ${ }^{11}$. L'union de Cicéron et de Terentia se terminera par un divorce (fin 47 ou début 46) ${ }^{12}$. Cependant, aucun signe de mésentente n'apparaît dans les lettres d'exil. Par contre, les lettres de l'année 57 font allusion, à mots couverts, à des difficultés avec Terentia ${ }^{13}$.

Nous savons que Tullia, l'aînée du couple, est née un 5 août: Cicéron, à son retour d'exil, a débarqué à Brindes le 5 août, jour anniversaire de sa fille ${ }^{14}$. Mais nous ignorons l'année exacte. Pour Pierre Grimal, ce serait le 5 août $76^{15}$. Tullia était déjà mariée en décembre 63, puisque Cicéron fait allusion à son gendre dans la $4^{\mathrm{e}}$ Catilinaire ( $(3)$. Si elle est née en 76, elle n'avait alors que 13 ans, mais, comme le rappelle Pierre Grimal, il était d'usage à Rome de marier les filles de très bonne heure. Le gendre de Cicéron s'appelle C. Calpurnius Piso Frugi. Il est de famille noble et apparenté au consul de 58, L. Calpurnius Piso Caesoninus. Plus tard, Cicéron fera de lui un bel éloge dans le Brutus (\$272), le présentant comme un des jeunes gens les plus doués de sa génération et louant aussi sa pietas.

7 Quant au fils de Cicéron et de Terentia, il était sensiblement plus jeune que sa sœur, puisqu'il est né en juillet 65 (sa date de naissance nous est fournie par une lettre de son père $\left.{ }^{16}\right)$. En 58 , c'était donc un enfant de 7 ans. Il porte les mêmes noms que son père : M. Tullius Cicero. Son père, dans ses lettres, l'appelle toujours Cicero.

8 Les quatre lettres d'exil à Terentia sont adressées à Terentia et à ses enfants. L'en-tête (inscriptio) est presque toujours le même :

- Fam. 14, 4 : Tullius s. d. Terentiae et Tulliae et Ciceroni suis.

- Fam. 14, 2 : Tullius s. d. Terentiae suae et Tulliolae et Ciceroni suis.

- Fam. 14, 1 : Tullius Terentiae suae, Tulliolae suae, Ciceroni suo salutem dicit.

- Fam. 14, 3 : Tullius s. d. Terentiae suae et Tulliae et Ciceroni. 
Cicéron se désigne par son nomen, Tullius, comme il le fait toujours dans les lettres à sa famille et à son esclave, puis affranchi, Tiron (assimilé à un membre de la famille). L'emploi du possessif souligne l'affection qui l'unit aux siens. La fille de Cicéron est tantôt désignée par le nom de Tullia, tantôt par le diminutif de Tulliola ${ }^{17}$.

Mais, en dépit de cet en-tête, l'essentiel de la lettre s'adresse à Terentia, comme le montre l'emploi de la deuxième personne du singulier. Le uos n'apparaît que dans quelques phrases au début et à la fin. Dans une des lettres, Cicéron dit même assez curieusement Tulliolae et Ciceroni salutem dic, comme s'ils n'étaient pas destinataires de la lettre ${ }^{18}$.

Mon projet est donc de comparer les lettres à Terentia avec les lettres à Atticus. Plutôt que de comparer lettre par lettre, j'ai préféré synthétiser les résultats de mon enquête. Je montrerai d'abord l'importance plus grande de l'émotion, de l'affectivité, dans les lettres à Terentia, ensuite la place plus grande qu'y tient la vie privée, la vie familiale. Cependant la vie publique, la vie politique, est loin d'être absente des lettres à Terentia et, de ce point de vue, elles ne sont pas tellement éloignées des lettres à Atticus ${ }^{19}$.

\section{Émotion et affectivité}

11 L'émotion se traduit d'abord par le fait de verser des larmes (lacrimae, fletus). Le thème des larmes apparaît dès le début de la première lettre à Terentia et il se retrouve dans toutes les lettres suivantes : Cicéron ne peut pas écrire aux siens ni lire leurs lettres sans verser des pleurs ${ }^{20}$. Certes les larmes ne sont pas absentes des lettres à Atticus : "Je ne dis rien de mes autres douleurs intolérables; car je suis arrêté par les larmes » y lisons-nous en date du 17 juin ${ }^{21}$. La situation cependant est assez différente : Cicéron pleure ici sur ses propres malheurs, il ne pleure pas parce qu'il écrit à Atticus. De ce point de vue, les lettres à Terentia sont plus proches des lettres à Quintus : en écrivant à son frère, Cicéron ne peut pas non plus retenir ses larmes ${ }^{22}$.

12 Autre marque d'émotion: les apostrophes dans le corps de la lettre, dont JacquesEmmanuel Bernard a souligné l'importance ${ }^{23}$. Ces apostrophes sont plus nombreuses et surtout plus riches et plus variées dans les lettres à Terentia que dans les lettres à Atticus, comme le montre le relevé suivant.

Dans les lettres ad suos :

- Fam. 14, 4, 1 : mea uita

- Fam. 14, 4, 5 : mea Terentia

- Fam. 14, 4, 6: mea Terentia, fidissima atque optima uxor, et mea karissima filiola et spes reliqua nostra, Cicero

- Fam. 14, 2, 2 : mea lux, meum desiderium [...] mea Terentia

- Fam. 14, 2, $3:$ mea uita

- Fam. 14, 2, 4 : mea desideria

- Fam. 14, 1, 5 ; 14, 3, 1; 14, 3, 5 : mea Terentia

Dans les lettres à Atticus :

- Att. 3, 4 ; 3, 9, 2 : mi Pomponi

- Att. 3, 15, 7 : Pomponi

- Att. 3, 19, 3 : T. Pomponi

- Att. 3, 22, 3 ; 3, 23, 2 : mi Pomponi 
Atticus est généralement appelé, dans les lettres d'exil, mi Pomponi ${ }^{24}$. Dans la lettre Att. 3,15 , où Cicéron lui fait des reproches, il est appelé plus sèchement Pomponi. Dans un passage plus solennel, Cicéron utilise le prénom et le nom de famille : Tite Pomponi. Dans le cas de Terentia et des enfants, la variété des appellations est nettement plus grande. Mea uita et mea lux évoquent la poésie amoureuse. Mea uita se trouve chez Catulle, Ovide et Properce, tandis qu'ailleurs Properce utilise simplement uita, au vocatif ${ }^{25}$. Mea lux, ou lux mea, apparaît dans le Corpus Tibullianum, chez Properce, dans les Amours et l'Art d'aimer d'Ovide ${ }^{26}$. Meum desiderium semble plus rare au vocatif ${ }^{27}$. Desiderium peut signifier " objet de regret» (L.-A. Constans traduit : "objet de mes tendres regrets »), mais également «objet d'amour " ${ }^{28}$. Le pluriel mea desideria englobe Terentia et les enfants ${ }^{29}$.

L'émotion se traduit enfin par l'illusion de la présence : Cicéron pense intensément à sa femme et à sa fille et croit les avoir devant lui, sous ses yeux. Cette illusion de présence fait jaillir les larmes ${ }^{30}$. Nous ne trouvons rien de tel dans les lettres à Atticus ni même dans les lettres à Quintus ${ }^{31}$. En revanche on peut découvrir des parallèles dans la poésie amoureuse : illum absens absentem auditque uidetque, écrit Virgile à propos de Didon amoureuse d'Énée ${ }^{32}$.

L'émotion éprouvée par Cicéron lorsqu'il écrit aux siens s'explique par le fait qu'eux aussi sont victimes de son exil : ils sont atteints à la fois dans leurs biens (la maison familiale a été pillée et détruite) et dans leur réputation. Ce n'est évidemment pas le cas d'Atticus. Par contre, c'est le cas de Quintus, dont la carrière politique risque de pâtir de l'exil de son frère. Bien plus, Cicéron s'estime responsable du malheur dans lequel sont plongés les siens. Cette idée n'apparaît pas dans la première lettre, où, pour réconforter Terentia, Cicéron affirme qu'ils n'ont commis aucune faute ${ }^{33}$. En revanche le sentiment de culpabilité et même de honte s'affirme dans les trois lettres suivantes. Ainsi dans la lettre du 5 octobre (Cic., Fam. 14, 2, 1):

Vos enim uideo esse miserrimas, quas ego beatissimas semper esse uolui, idque praestare debui et, nisi tam timidi fuissemus, praestitissem ${ }^{34}$.

Je vois en effet que vous êtes très malheureuses, vous que j'ai toujours voulues parfaitement heureuses, à qui j'aurais dû assurer ce bonheur et à qui je l'aurais assuré si je n'avais pas été si timoré.

Cicéron se reproche de n'avoir pas cherché à lutter et d'être parti de lui-même pour l'exil avant le vote de la première loi de Clodius. Il estime que ce manque de courage a causé le malheur de sa famille. Tout cela explique le bouleversement qu'il éprouve lorsqu'il écrit aux siens ou reçoit leurs lettres.

\section{Vie privée et vie familiale}

Nous en venons maintenant à la place qu'occupe la vie privée dans les lettres à Terentia. Dans les lettres à Atticus, Cicéron mentionne régulièrement les siens (mei) ou ses enfants (liberi). Il les recommande à Atticus ou exprime sa douleur d'être séparé d'eux ${ }^{35}$. Mais, en règle générale, il ne parle pas de chaque enfant pris individuellement (même si le nom de Tullia et celui de "Cicero" apparaissent chacun une fois ${ }^{36}$ ). En revanche, dans les lettres à Terentia (ou du moins dans deux d'entre elles), Cicéron consacre quelques lignes à chaque membre de la famille. Ainsi, dans la lettre envoyée de Brindes, Cicéron a d'abord parlé de Terentia, en s'inquiétant pour sa santé comme il le fait souvent ${ }^{37}$. Il en vient ensuite à Tullia (Cic., Fam. 14, 4, 3) : 
Sed quid Tulliola mea fiet? Iam id uos uidete; mihi deest consilium. Sed certe, quoquo modo se res habebit, illius misellae et matrimonio et famae seruiendum est.

Mais qu'adviendra-t-il de ma chère petite Tullia ? Désormais c'est à vous de voir, car l'esprit de décision me fait défaut. En tout cas, de quelque façon que les choses se présentent, il faut tout faire pour préserver le mariage et la réputation de cette pauvre petite.

Selon L.-A. Constans, le passage ferait allusion à la dot de Tullia, qui n'aurait pas été intégralement versée: Cicéron, dont les biens ont été confisqués, ne serait pas en mesure d'en achever le versement ${ }^{38}$. Cette hypothèse est écartée par Susan Treggiari : pour elle, si le mariage a eu lieu en 63 , en 58 la dot devait déjà être versée ${ }^{39}$. Ce que redoute alors Cicéron, c'est probablement que Pison demande le divorce. Il est engagé dans le cursus honorum et exerce, en 58 , la fonction de questeur ${ }^{40}$. Il pourrait être tenté de se défaire d'une alliance qui risque désormais de nuire à sa carrière ${ }^{41}$. Après avoir ainsi exprimé son inquiétude pour Tullia, Cicéron passe à son fils, avant de revenir à Terentia.

Les enfants de Cicéron sont évoqués aussi avec précision dans la lettre à Terentia du 25 novembre $(F a m .14,1,1)$ et dans une des deux lettres à Quintus (Q. fr. 1, 3, 3). Dans ce dernier passage, Cicéron ajoute la mention d'un autre enfant, son neveu, le fils de son frère, né en $67^{42}$.

Un autre membre de la famille apparaît régulièrement dans les lettres à Terentia : c'est le mari de Tullia, Pison. Bien loin de demander le divorce, celui-ci se montre un gendre loyal et travaille activement au rappel de l'exilé. Dans les lettres à Atticus et à Quintus, il n'est mentionné qu'incidemment ${ }^{43}$. Dans les lettres à Terentia, Cicéron fait régulièrement son éloge ${ }^{44}$ : «Les dieux fassent qu'il me soit permis de jouir de la compagnie d'un tel gendre, avec toi et avec nos enfants!", écrit-il le 29 novembre ${ }^{45}$. Ce souhait ne se réalisera pas, car Pison va mourir au cours du premier semestre de 57. C'est la période pour laquelle nous n'avons pas de lettre de Cicéron: nous ignorons donc la date exacte et les causes de son décès. La plus claire allusion à la mort de Pison se trouve dans le Pro Sestio: «Pison mon gendre, qui n'a pu recevoir ni de moi ni du peuple romain la récompense de son dévouement $[. . .]^{46} »$.

21 Autre aspect de la vie familiale présent dans les lettres : tout ce qui concerne les biens, les propriétés de la famille. Il y a ainsi la question des esclaves, abordée dans la lettre de Brindes. Comme les autres biens de Cicéron, les esclaves vont être vendus aux enchères. Leur maître les a affranchis sous condition, pour le cas où ils ne pourraient pas être rachetés par l'un de ses proches ${ }^{47}$. Cicéron s'inquiète aussi pour la fortune personnelle de Terentia: même si elle n'a pas été confisquée, elle risque cependant d'être mise à mal. Terentia doit accepter l'aide financière de leurs amis, elle doit conserver ses richesses dans l'intérêt de leur fils ${ }^{48}$. Le bien auquel Cicéron tient le plus est manifestement sa maison du Palatin: c'est pourquoi il en parle non seulement à Terentia, mais aussi à Atticus ${ }^{49}$.

Je signalerai enfin quelques passages de la lettre de Brindes intéressants du point de vue de la vie privée. Il y a d'abord la mention des dieux : "ni les dieux, que tu as honorés avec tant de piété, ni les hommes, que j'ai toujours servis, ne nous ont témoigné de reconnaissance ${ }^{50}$ ». Elle est d'autant plus intéressante que les références religieuses sont rares dans la correspondance. Les lettres d'exil à Atticus ne contiennent aucune allusion aux dieux, peut-être parce qu'Atticus était épicurien : pour lui les dieux n'interviennent pas dans les affaires humaines et ne peuvent donc apporter aucun secours à l'exilé Cicéron. En revanche les dieux sont mentionnés dans la 
première lettre à Quintus : Cicéron affirme qu'il les prie, bien qu'ils soient devenus sourds à ses prières ${ }^{51}$.

Plus loin dans sa lettre à Terentia (\$ 2), Cicéron raconte qu'il est resté treize jours chez un certain M. Laenius Flaccus, qui n'a pas hésité à la recevoir en dépit des sanctions prévues par la loi de Clodius ${ }^{52}$. Dans la lettre qu'il écrit le même jour à Atticus (Att. 3, 7), Cicéron ne juge pas nécessaire de parler de son hôte de Brindes. L'important pour lui est que la tradition familiale garde le souvenir de l'action généreuse accomplie par M. Laenius Flaccus. Si lui-même ne peut pas lui témoigner sa reconnaissance, sa famille, son fils en particulier, pourra peut-être le faire un jour.

Enfin, dans le dernier paragraphe de la lettre (\$ 6), Cicéron parle à Terentia de quatre amis qui l'ont accompagné jusqu'à Brindes, alors qu'il ne les mentionne pas dans sa lettre à Atticus. Sans doute s'agit-il d'amis de rang modeste, de clients, qui étaient peu connus du grand personnage qu'est Atticus ${ }^{53}$. En revanche, ils devaient fréquenter la domus du Palatin et être pour Terentia des figures familières. D'autre part, cette dernière semble avoir été plus attentive qu'Atticus aux conditions de vie de l'exilé. Ainsi nous voyons Cicéron la rassurer à propos d'une épidémie qui sévissait à Thessalonique ${ }^{54}$. Il n'est nulle part question de cette pestilentia dans les lettres à Atticus, qu'elle ne devait pas inquiéter outre mesure.

\section{Vie publique et vie politique}

Nous allons voir maintenant que la vie politique est loin d'être absente des lettres à Terentia. Nous pouvons d'abord constater que Terentia elle-même suit la vie politique romaine. Cicéron lui écrit : "Je vois que tu mets ton espoir dans les nouveaux tribuns de la plèbe ${ }^{55}$.» Les nouveaux tribuns de la plèbe ont été élus en juillet et prendront leurs fonctions le 10 décembre. On pense au début qu'ils seront tous les dix favorables à Cicéron. Terentia le sait et a écrit à ce sujet à son mari pour le rassurer et l'encourager.

Cicéron, de son côté, lui parle de la situation politique. Ainsi le 25 novembre : «Mais cependant, si nous avons avec nous tous les tribuns de la plèbe, et Lentulus, aussi dévoué qu'il paraît l'être, et encore Pompée et César, il ne faut pas désespérer ${ }^{56}$. Un peu plus haut dans la même lettre il est revenu sur les événements des mois écoulés : «Tout cela est arrivé par ma faute; je pensais que j'étais aimé de ceux qui me jalousaient et je ne suivais pas ceux qui me recherchaient ${ }^{57}$. " L'expression qui petebant, " ceux qui me recherchaient ", désigne principalement César ${ }^{58}$. Cicéron écrit en effet dans la lettre suivante : «Mon devoir était soit d'échapper au danger en acceptant une légation, soit de résister activement et avec tous mes moyens, soit de succomber courageusement ${ }^{59}$.» En juillet 59, César, alors consul, avait proposé à Cicéron de le prendre comme légat l'année suivante, lors de son proconsulat en Gaule ${ }^{60}$. S'il avait accepté, Cicéron se serait inféodé à César, mais il aurait échappé aux menaces de Clodius. Il regrette maintenant de ne pas avoir accepté la legatio, estimant que c'était son devoir (officium) envers les siens. C'est un détail intéressant, sur le plan politique, qui ne figure pas dans les lettres à Atticus.

Terentia n'est pas seulement spectatrice de la vie politique romaine; les lettres de Cicéron nous montrent qu'elle travaille activement en faveur du retour de l'exilé ${ }^{61}$. Pour cela elle fait preuve de qualités toutes masculines : uirtus, fortitudo ${ }^{62}$, ces qualités qui ont fait défaut à son mari ${ }^{63}$. En quoi peut consister son action? Selon Susan Treggiari, ce serait des démarches soit auprès de femmes influentes, comme Julia, fille 
de César et femme de Pompée, soit directement auprès d'hommes influents, comme Pompée ou les consuls désignés, Lentulus et Metellus ${ }^{64}$.

Terentia serait prête à rejoindre son mari dans son exil. Mais celui-ci estime que sa présence est plus utile à Rome ${ }^{65}$. Cela correspond à ce que Cicéron avait écrit quelques mois plus tôt à Atticus ${ }^{66}$. Atticus, lui aussi, travaille assidûment au retour de l'exilé.

Cicéron demande à sa femme de lui écrire ${ }^{67}$. Il est un cas où il lui demande clairement de l'informer sur la situation politique (Cic., Fam. 14, 3, 3-4) :

Nunc spes reliqua est in nouis tr. pl. et in primis quidem diebus; nam si inueterarit, actum est. Ea re ad te statim Aristocritum misi ut ad me continuo initia rerum et rationem totius negotii posses scribere ${ }^{68}$.

Maintenant ce qui me reste d'espoir est dans les nouveaux tribuns de la plèbe, et encore dans les premiers jours de leur magistrature; car si elle perd de sa nouveauté, c'en est fini. Je te renvoie donc tout de suite Aristocrite afin que tu puisses m'écrire sans retard comment les choses ont commencé et comment se déroule toute l'affaire.

30 Terentia doit écrire ce que font les tribuns dans les premiers jours de leur tribunat. Cicéron adresse la même demande à Atticus à la fin de la lettre qu'il lui envoie le même jour ${ }^{69}$. L'ami et l'épouse remplissent le même rôle d'informateur politique.

De son côté, Terentia demande à Cicéron d'écrire à certaines personnes pour les remercier ${ }^{70}$. S'agit-il d'amis qui soutiennent financièrement la famille, ou de personnalités que Terentia a sollicitées en faveur de l'exilé? Il nous est difficile de répondre à cette question.

Terentia, qui se dévoue aux intérêts de son mari et de sa famille, est victime un jour d'un incident très mystérieux pour nous (Cic., Fam. 14, 2, 2):

Maereo casum eius modi ut tantis tuis miseriis meae miseriae subleventur. Nam ad me P. Valerius, homo officiosus, scripsit, id quod ego maximo cum fletu legi, quemadmodum a Vestae ad tabulam Valeriam ducta esses. Hem, mea lux, meum desiderium, unde omnes opem petere solebant! te nunc, mea Terentia, sic uexari, sic iacere in lacrimis et sordibus, idque fieri mea culpa, qui ceteros seruaui, ut nos periremus ${ }^{71}$ !

Je déplore que mon malheur soit tel que tu doives, pour soulager mes misères, éprouver toi-même de si grandes misères. Car P. Valerius, un homme très dévoué, m'a écrit - et je n'ai pu le lire sans verser bien des larmes - comment tu avais été conduite du temple de Vesta à la tabula Valeria. Hélas! toi qui es ma lumière, toi qui me manques tant, tous avaient coutume de demander ton aide : faut-il maintenant, ma chère Terentia, que tu sois ainsi maltraitée, que tu sois plongée dans les larmes et le deuil, et que tout cela arrive par ma faute, à moi qui ai sauvé tous les autres pour nous conduire, nous, à notre perte !

Le passage, très allusif, est loin d'être clair. La tabula Valeria semble avoir été non pas une banque, comme le pensait L.-A. Constans ${ }^{72}$, mais une peinture murale commémorant la victoire du consul Manius Valerius Maximus Messala, remportée en 263 sur les Carthaginois et Hiéron de Syracuse (c'est un épisode de la première guerre punique) ${ }^{73}$. Près de cette peinture se réunissaient les tribuns de la plèbe. Terentia aurait donc été conduite, au milieu de manifestations hostiles, non pas à la vente aux enchères des biens de son mari, mais devant les tribuns de la plèbe (Clodius ?), peut-être pour y subir un interrogatoire ${ }^{74}$. Pourquoi le temple de Vesta? On a parfois supposé qu'après la destruction de la maison du Palatin, Terentia s'était réfugiée dans la maison des Vestales, auprès de sa demi-sœur Fabia. Cette hypothèse, que L.-A. Constans juge inutile, n'est pas écartée par Susan Treggiari ${ }^{75}$. Il n'est pas possible de parvenir à une certitude sur ce point. Il faut noter que Cicéron a été mis au 
courant de l'incident, non pas par Terentia elle-même, mais par un de ses amis, P. Valerius ${ }^{76}$. Il semble que Terentia n'ait pas voulu raconter à son mari un incident qui ne pouvait qu'accroître son désarroi et son sentiment de culpabilité.

\section{Conclusion}

Nous avons vu que les lettres à Terentia se distinguent des lettres à Atticus par la place plus grande accordée à l'émotion et à la vie familiale. On peut ajouter un autre point : l'absence de reproches. Atticus, dans ses lettres, critiquait Cicéron pour sa faiblesse et son manque de courage face à l'épreuve ${ }^{77}$. Cicéron, lui, reproche à Atticus de n'avoir pas su le conseiller au moment décisif ${ }^{78}$. Par contre il semble n'avoir rien à reprocher à Terentia. Celle-ci, de son côté, voit dans le malheur qui frappe sa famille l'œuvre du destin, et non pas la conséquence de fautes commises par son mari ${ }^{79}$.

Cependant les lettres à Atticus et les lettres à Terentia se rejoignent dans la mesure où tous deux sont les soutiens de Cicéron et préparent activement son retour. On notera encore qu'Atticus n'est jamais mentionné dans les lettres d'exil à Terentia ${ }^{80}$. Cicéron n'éprouve pas le besoin de parler de son ami à sa femme. Il sait qu'à Rome tous deux sont proches et travaillent de concert à une œuvre commune : le salut (salus) de l'exilé 81 .

\section{BIBLIOGRAPHIE}

\section{Textes anciens}

Cicéron, Correspondance, II, texte établi et trad. par L.-A. Constans, CUF, Paris, 1935.

Cicéron, Correspondance, V, texte établi et trad. par J. Bayet, CUF, Paris, 1964.

Cicéron, Correspondance, VI, texte établi, trad. et annoté par J. Beaujeu, CUF, Paris, 1993.

Cicero, Letters to Atticus, II, ed. by D. R. Shackleton Bailey, Cambridge Classical Texts and

Commentaries 4, Cambridge, 1965.

Cicero, Epistulae ad familiares, I, ed. by D. R. Shackleton Bailey, Cambridge Classical Texts and Commentaries 17, Cambridge, 1977.

\section{Textes modernes}

BARDT C. 1913, Römische Charakterköpfe in Briefen, Leipzig.

BEARD M. 2002, « Ciceronian Correspondences: Making a Book out of Letters », in T. P. Wiseman (éd.), Classics in Progress, Essays on Ancient Greece and Rome, British Academy Centenary Monographs, Oxford, p. 103-144. 
BERNARD J.-E. 2013, La sociabilité épistolaire chez Cicéron, Babeliana 16, Paris.

CARCOPINO J. 1947, Les secrets de la correspondance de Cicéron, Paris, 2 vol.

CLAASSEN J. M. 1996, «Documents of a Crumbling Marriage: The Case of Cicero and Terentia », Phoenix 50, p. 208-232.

COARELLI F. 1999, « Tabula Valeria », in E. M. Steinby (éd.), Lexicon topographicum Vrbis Romae, V, Rome, p. 16.

DIXON S. 1986, « Family Finances: Terentia and Tullia », in B. Rawson (éd.), The Family in Ancient Rome. New Perspectives, Londres - Sidney, p. 93-120.

GARCEA A. 2005, Cicerone in esilio. L'epistolario e le passioni, Spudasmata 103, Hildesheim - New York.

GAVoILLE É. 2000, «La relation à l'absent dans les lettres de Cicéron à Atticus », in L. Nadjo,

É. Gavoille (éds), Epistulae antiquae. Actes du $I^{e r}$ colloque « Le genre épistolaire antique et ses

prolongements ", Université François-Rabelais, Tours, 18-19 septembre 1998, Louvain - Paris, p. 153-176.

GREBE S. 2003, « Marriage and Exile: Cicero's Letters to Terentia », Helios 30, p. 127-146.

GRIMAL P. 1986, Cicéron, Paris.

HUTCHINSON G. O. 1998, Cicero's Correspondence. A Literary Study, Oxford.

JEPPESON-WIGELSWORTH A. 2013, « Amici and Coniuges in Cicero's Letters: Atticus and Terentia », Latomus 72, p. 350-365.

RAWSON E. 1994, Cicero. A Portrait, $3^{\text {e }}$ éd., Londres [1 ${ }^{\text {re }}$ éd. : 1975].

TREGGiaRi S. 2007, Terentia, Tullia and Publilia: The Women of Cicero's Family, Women of the Ancient World, Londres - New York.

\section{NOTES DE FIN}

1. Voir Cic., Att. 3, 8, 4;3, 9, $3 ;$ Fam. 14, 1, 5; 14, 3, 3. Fam. 14, 1, 4, se réfère à une lettre à Terentia aujourd'hui perdue. Je cite les lettres de Cicéron d'après l'édition de la CUF. Les traductions sont personnelles.

2. Constans (éd.) 1935, p. 26 et 92, n. 1. Shackleton Bailey (éd.) 1965, p. 165, observe que la lettre Att. 4, 1, 2, sonne un peu étrangement si Atticus et Cicéron ont été ensemble de février à juillet. L'hypothèse de Constans lui paraît cependant plus vraisemblable que celle de Bardt 1913, p. 173, n. 1, selon laquelle Atticus aurait supprimé les lettres de cette période parce qu'elles révélaient une brouille entre Cicéron et lui. Beard 2002, p. 125-127, a ouvert sur la question de nouvelles perspectives: selon elle, les lettres ont pu être sélectionnées et agencées par les premiers éditeurs de façon à construire une œuvre littéraire ("making a book out of letters»); ainsi la dernière lettre du livre 3 fait écho à la première («ring-composition») et forme un contraste ironique avec le début du livre 4.

3. Selon Carcopino 1947, II, p. 436-438, les lettres de cette période auraient toutes été supprimées car elles relataient les transactions précédant le retour de Cicéron et donnaient une mauvaise image de César (rappelons que, pour Carcopino, la correspondance de Cicéron a été publiée dans le but de servir la propagande d'Octave, fils adoptif de César). À mes yeux, l'hypothèse d'une perte accidentelle demeure cependant la plus vraisemblable.

4. Sur cette lettre, voir Hutchinson 1998, p. 28-33.

5. Voir Shackleton Bailey (éd.) 1977, p. 289.

6. Cic. Att. 3, 9, et Q. fr. 1, 3. 
7. Voir Treggiari 2007, p. 27.

8. Grimal 1986, p. 67.

9. Plu., Cic. 8, 3.

10. Voir par ex. Cic., Fam. 14, 1, 5. Voir aussi Att. 2, 4, 5.

11. Voir Plu., Cat. Mi. 19, 5 ; Asc., 91C. Cf. Sal., Cat. 15, 1.

12. Voir Treggiari 2007, p. 129. Sur ce divorce, voir Claassen 1996.

13. Cic., Att. 4, 1,$8 ; 4,2,7$.

14. Cic., Att. 4, 1, 4 ; Sest. 131.

15. Grimal 1986, p. 67.

16. Cic., Att. 1, 2, 1.

17. Sur l'en-tête ou adresse des lettres (inscriptio), voir Bernard 2013, p. 133-138.

18. Cic., Fam. 14, 1, $6:$ « Salue de ma part Tulliola et Cicéron ».

19. Sur les similitudes que présentent, de façon générale, les lettres à Atticus et les lettres à Terentia, voir Jeppesen-Wigelsworth 2013.

20. Cic., Fam. 14, 4, 1 : cum aut scribo ad uos aut uestras lego, conficior lacrimis sic ut ferre non possim, «quand je vous écris ou que je lis vos lettres, je suis accablé de larmes à un point que je ne puis supporter ». Voir aussi Cic., Fam. 14, 2, 1; 14, 2, 2; 14, 1, 5; 14, 3, 1; 14, 3, 5.

21. Cic., Att. 3, 10, 2 : Mitto cetera intolerabilia ; etenim fletu impedior.

22. Cic., Q. fr. 1, 3, 3 : Haec ipsa me quo fletu putas scripsisse? Eodem quo te legere certe scio. An ego possum aut non cogitare aliquando de te aut umquam sine lacrimis cogitare?, "Ces mots mêmes, imagines-tu avec quels pleurs je les ai écrits? Les mêmes pleurs avec lesquels, je le sais bien, tu les lis. Puis-je rester un instant sans penser à toi ou y penser jamais sans verser des larmes?». Voir aussi Q. fr. 1, 3, 10.

23. Bernard 2013, p.139-141. Sur les apostrophes dans les lettres d'exil, voir Garcea 2005, p. 126-128.

24. Cicéron ne tient pas compte de l'adoption d'Atticus par son oncle Q. Caecilius, sauf dans l'entête de Att. 3, 20.

25. Catul., 45, 13 ; 109, 1 ; Ov., Am. 2, 15, 21 ; Prop., 2, 3, 23 ; 2, 26, 1. Vita : Prop., 1, 2, 1 ; 2, 24, 29. Chez Plaute, on trouve déjà mea uita ou o mea uita, généralement en contexte amoureux (Cas. 135 ; Poen. 365 ; Truc. 391), mais aussi dans la bouche d'un parasite (St. 584).

26. Corpus Tibullianum (Sulpicia) 3, 9, 15 ; Prop., 2, 14, 29 ; 2, 28, 59 ; II, 29, 1 ; Ov., Am. 1, 4, 25 ; Ars 3 , 524. Chez Catulle lux mea se trouve au nominatif $(68,132$ et 162$)$.

27. Voir cependant Petr., 139, 4.

28. Voir Catul., 2, 5.

29. Dans les lettres d'exil à Quintus, il faut signaler le triple mi frater qui ouvre, de façon saisissante, Q. fr. 1, 3. Voir aussi Cic., Q. fr. 1, 3, $10 ; 1,4,1:$ mi frater.

30. Voir Cic., Fam. 14, 2, 3 : Nam mihi ante oculos dies noctesque uersaris; omnis labores te excipere uideo ;

timeo

sustineas, «Car jour et nuit tu es présente devant mes yeux; je vois que tu affrontes toutes les fatigues ; je crains que tu ne puisses pas tenir bon »; Fam. 14, 3, 2, où l'on retrouve les expressions dies noctesque et ante oculos uersari ; Fam. 14, 3, 5.

31. Il est vrai qu'en septembre 51, Cicéron, alors en Cilicie, écrira à Atticus : Video enim te [...] (Att. 5, 18, 3). Voir sur ce passage, Gavoille 2000, p. 171-172.

32. Verg., A. 4, 83 : «Absente, absent, elle l'entend et le voit». Comparer Ov., Ep. 7, 26 : Aenean animo noxque diesque refert, «La nuit, le jour ramènent Énée dans mes pensées ».

33. Cic., Fam. 14, $4,5$.

34. Voir aussi Cic., Fam. 14, 2, 2; 14, 1, 1; 14, 3, 1-2. Sur la responsabilité de Cicéron dans le malheur des siens, voir Q. fr. 1, 4,1;1, 4, $4 ;$ Att. 3, 15, $7 ; 3,19,3$.

35. Recommandation des siens : Att. 3, 6; 3, 19, 3 ; 3, 27. Douleur de la séparation : Att. 3, 10, 2 ; 3 , $15,2$. 
36. Cic., Att. 3, 19, 2 ; 3, 23, 5.

37. Voir Cic., Fam. 14, 2, 3 : ualetudinem istam infirmam ; Fam. 14, 3, 2 : infirmitas ualetudinis tuae.

38. Constans (éd.) 1935, p. 16. Voir aussi Shackleton Bailey (éd.) 1977, p. 286.

39. Treggiari 2007, p. 68. L'usage semble avoir été de faire trois versements, chaque fois à un an d'intervalle.

40. Cic., Red. Sen. 38.

41. Voir ce que Cicéron écrit au $\S 4$ : Pisonem, ut scribis, spero fore semper nostrum, «Quant à Pison, j'espère, comme tu l'écris, qu'il sera toujours avec nous ".

42. À cette époque, quand il s'agit de parler de ses proches, Cicéron se montre plus réservé dans les lettres à Atticus que dans les lettres à Terentia et à Quintus. Les choses changeront par la suite, les deux amis ayant progressé dans l'intimité l'un de l'autre ; voir par ex. Att. 10, 8, 9; 11, $17 ; 11,25,3$.

43. Cic., Q. fr. 1, 4, 2 ; Att. 3, 22,1 .

44. Cic., Fam. 14, 2, $2 ; 14,1,4 ; 14,3$, 3. Il faut se souvenir que les lettres sont adressées aussi à Tullia.

45. Cic., Fam. 14, 3, 3 : Di faxint ut tali genero mihi praesenti tecum simul et cum liberis nostris frui liceat!

46. Cic., Sest. 68 : Piso ille, gener meus, cui fructum pietatis suae neque ex me neque a populo Romano ferre licuit [...]. S'il avait vécu, le peuple romain aurait pu récompenser Pison en lui confiant une nouvelle magistrature.

47. Cic., Fam. 14, 4, 4. Cf. 14, 1, 3 .

48. Cic., Fam. 14, 2, 3 ; 14, 1, 5. Selon Dixon 1986, il y avait incertitude quant aux biens relevant de la dot de Terentia : Cicéron pouvait craindre qu'ils ne soient confisqués; les esclaves qualifiés de tui $($ Fam. 14, 4, 4) étaient une partie de la dot de Terentia.

49. Cic., Fam. 14, 2, 3 ; Att. 3, 15, 6 ; 3, 20, 2.

50. Cic., Fam. 14, 4, 1 : neque dii, quos tu castissime coluisti, neque homines, quibus ego semper seruiui, nobis gratiam rettulerunt. Même si Terentia, en tant que femme de magistrat, a joué un rôle dans la religion publique (voir Plu., Cic. 20,1-3), il est probable que Cicéron pense avant tout à la piété qu'elle manifestait dans le culte domestique. Voir Fam.14, 7, 1, où Cicéron lui demande de remercier le dieu qui l'a guéri quemadmodum soles, « comme tu en as l'habitude ».

51. Cic., Q. fr. 1, 3, 9 .

52. Sur l'accueil de M. Laenius Flaccus, voir Cic., Sest. 131 ; Planc. 97.

53. Sallustius, il est vrai, semble avoir été connu d'Atticus : voir Att. 1, 11, 1; 1, 3, 3. Sicca est mentionné en Att. 3, 2 et 3, 4, mais rien n'indique qu'il ait été bien connu d'Atticus à cette date. Pescennius et Clodius Philhetaerus (un affranchi ou fils d'affranchi) ne sont jamais nommés ailleurs dans la correspondance. Treggiari 2007, p. 64, qualifie ces quatre personnages de « humbler friends ». Sallustius, l'ami de Cicéron, est à distinguer de Sallustius, questeur en 50, destinataire de Fam.2, 17 (voir sur ce point Shackleton Bailey [éd.]1977, p. 457; contra, Constans [éd.] 1935, p. 17, n. 2). Tous deux sont à distinguer de l'historien Salluste.

54. Cic., Fam. 14, $1,3$.

55. Cic., Fam. 14, 2, 2 : In nouis tr. pl. intellego spem te habere.

56. Cic., Fam. 14, 1, 2 : sed tamen si omnis tr. pl. habemus, si Lentulum tam studiosum quam uidetur, si uero etiam Pompeium et Caesarem, non est desperandum. Voir aussi Fam. 14, 2, 2 ; 14, 3, 3.

57. Cic., Fam. 14, 1, 1 : sed omnia sunt mea culpa commissa, qui ab iis me amari putabam qui inuidebant, eos non sequebar qui petebant.

58. Voir sur ce point Shackleton Bailey (éd.) 1977, I, p. 289. Sur les amis envieux, qui par jalousie auraient conseillé à Cicéron de quitter Rome, voir Att. 3, 7, 2; 3, 9, 2; 3, 15, 2; 4, 3, 5 ; Q. fr. 1, 4, 1. Cf. Q. fr. 1, 4, 2; Fam. 14, 3, 2. Cicéron incrimine surtout le grand orateur Hortensius: Att. 3, 9, 2 ; Q. fr. $1,3,8$. 
59. Cic., Fam. 14, 3, 1 : Meum fuit officium uel legatione uitare periculum uel diligentia et copiis resistere uel cadere fortiter.

60. Voir Att. 2, 18, 3 ; 2, 19, 5. Selon Att. 2, 19, 4, Cicéron aurait également pu faire partie de la commission chargée de faire appliquer la loi agraire de César.

61. Voir Cic., Fam. 14, 4, 3 : si est spes nostri reditus, eam confirmes et rem adiuues, «s'il y a quelque espoir que je puisse rentrer, consolide-le et favorise la chose »;14, 2, 2 et 3; 14, 1, 2 : tuo labori, «tes efforts» (en faveur du retour de Cicéron). Grebe 2003 souligne le fait que c'est l'exil de son mari qui amène Terentia à jouer un rôle politique que normalement les femmes romaines ne jouaient pas.

62. Cic., Fam. 14, $1,1$.

63. Cic., Fam. 14, 3, 2 : Cicéron a manqué de uirtus et de diligentia au service des siens.

64. Treggiari 2007, p. 66.

65. Cic., Fam. 14, 3, 5 : Quod scribis te, si uelim, ad me uenturam, ego uero, cum sciam magnam partem istius oneris abs te sustineri, te istic esse uolo, "Tu écris que, si je le souhaite, tu viendras me rejoindre ; mais, sachant qu'une grande partie de cette charge repose sur toi, je souhaite que tu restes là-bas »). Voir déjà Fam. 14, 4, 3. Cf. Q. fr. 1, 3, 3.

66. Cic., Att. 3, 12, 3, du 17 juillet, avec emploi du même adverbe, istic.

67. Voir Cic., Fam. 14, 2, 4; 14, 1, 6 ; 14, 3, 5.

68. Aristocrite est le nom d'un courrier (voir Cic., Fam. 14, 3, 1).

69. Cic., Att. 3, 23, 5 .

70. Cic., Fam. 14, 1,$5 ; 14,3$, 3. Cf. 14, 2 , 4 .

71. Dans les discours Dom. 59 et Sest.54, Cicéron reproche à ses ennemis d'avoir maltraité Terentia (il emploie, comme ici, le verbe uexare). Voir aussi Sest. 145 ; Mil. 87.

72. Constans (éd.) 1935, p. 17 et 66.

73. Voir Coarelli 1999, p. 16. Cette interprétation se fonde sur Plin., Nat. 35, 22, et Cic., Vat. 21 (avec le commentaire du scholiaste de Bobbio).

74. Voir Shackleton Bailey (éd.) 1977, p. 287-288 ; Treggiari 2007, p. 65-66.

75. Constans (éd.) 1935, p. 17, n. 1 ; Treggiari 2007, p. 31 et 61. Dans le même sens que Treggiari, voir Shackleton Bailey (éd.) 1977, p. 287 ; Garcea 2005, p. 72.

76. Voir sur ce point Treggiari 2007, p. 65. Un P. Valerius, ami et hôte de Cicéron, est mentionné aussi en Cic., Phil. 1, 8, et en Att. 16, 7, 1. Voir aussi Att. 12, 51, 1 ; 12, 53 ; 13, 15.

77. Cic., Att. 3, 10, 2; 3, 10, 3; 3, 11, 2;3, 12, 1; 3, 13, 2;3, 15, 1; 3, 15, 7. Il y a peut-être une allusion à ces reproches en Dom. 97, où Cicéron dit que certains le trouvaient trop abattu.

78. Cic., Att. 3, 15, 4 ; 3, 15, 7; 4, 1, 1 .

79. Voir Cic., Fam. 14, 1, 1.

80. Par contre Terentia est nommée dans les lettres d'exil à Atticus. Cicéron la recommande à son ami (Att. 3, 19, 3; 3, 23, 5) ou fait part de ses remerciements $(3,5 ; 3,8,4 ; 3,9,3)$.

81. Cicéron emploie régulièrement le mot salus à propos de son retour d'exil. Voir Fam. 14, 4, 5 ; 14, 3, 2 ; Q. fr. 1, 3, $10 ; 1,4,2$; Att. 3, 19, 1; 3, 19, 2; 3, 20, 2; 3, 20, 3; 3, 23, 1; 3, 25 ; Fam. 1, 5, 1. Voir encore, en l'année 51, Fam. 15, $7 ; 15,8$.

\section{RÉSUMÉS}

Le tome II de la Correspondance contient quatre lettres de Cicéron à sa femme et à ses enfants (Fam. 14, 1-4). Ces lettres, envoyées pendant l'exil (58 av. J.-C.), s'adressent pour l'essentiel à l'épouse, Terentia. Pour chacune d'entre elles, nous disposons d'une lettre à Atticus écrite le même jour. La comparaison de ces textes permet de montrer comment l'épistolier adapte son discours à son destinataire, ami ou épouse. Les lettres à Terentia accordent une place plus grande à l'émotion et à la vie familiale. Cependant la vie politique de Rome est loin d'en être absente. 
The collection of letters Ad Familiares includes four letters Cicero has sent to his family during his exile (Fam. 14, 1-4, of 58 B.C.). They are mainly addressed to his wife, Terentia. Compared with the letters Cicero wrote at the same time to his friend Atticus, the letters to Terentia lay a major stress on emotion and private life. Nevertheless they are far from ignoring Roman politics and public life.

\section{INDEX}

nomsmotscles Asconius Pedianus, Catulle, César, Cicéron, Corpus Tibullianum, Ovide, Pétrone, Plaute, Pline l'Ancien, Plutarque, Properce, Salluste, Virgile

Keywords : Terentia, exile, private life, public life

Mots-clés : Terentia, exil, vie privée, vie publique

\section{AUTEUR}

\section{FRANÇOIS GUILLAUMONT}

Université de Tours, ICD (EA 6297) 